\title{
State Estimation in Low-Observable Distribution Systems Using Matrix Completion
}

\author{
Yingchen Zhang \\ NREL \\ yingchen.zhang@nrel.gov
}

\author{
Andrey Bernstein \\ NREL \\ andrey.bernstein@nrel.gov
}

\author{
Andreas Schmitt \\ Virginia Tech \\ andreas9@vt.edu
}

\author{
Rui Yang \\ NREL \\ rui.yang@nrel.gov
}

\begin{abstract}
The need for distribution system state estimation is on the rise because of the increased penetration of distributed energy resources and flexible load. To manage the distribution systems in real time, operators need to firstly overcome the challenge of low observability in distribution systems. Also, because of the amount of data present from smart meters, distributed generation measurements, switches, etc., the ideal distribution state estimation methods need to be able to process heterogeneous data.

In this paper, an algorithm is developed for voltage phasor estimation in low-observability distribution systems. The algorithm is based on the matrix completion approach from signal processing. The traditional matrix completion formulation is augmented with power-flow constraints to improve results while requiring less data. This method can also use all types of measurements (voltage magnitude, voltage angle, real power, reactive power) to complete the state matrix.
\end{abstract}

\section{Introduction}

Power systems state estimation (SE) is a long-established tool for bulk grid operation. It provides full visibility and the best estimate of the entire network status. It forms the foundational block for power system energy management applications, such as real-time power flow, real-time dispatch, and stability applications [1]. SE has not been broadly used in distribution system operation largely because traditionally distribution management systems are a collection of individual applications that do not require visibility of the entire system. Traditionally, power has flowed from the transmission grid to distribution systems and there were nearly no resources or controllable devices to manage in real time.

However, distribution systems are undergoing dramatic changes because of the fast adoption of the distributed energy resources (DERs), especially distributed photovoltaic (PV) systems. According to the SEIA report [2], the U.S. market recently installed 10.6 GW of solar PV and it reached 55.9
GW of total installed capacity (at the end of Q1 2018). For the new installations, $59 \%$ of the installed capacity came from the utility PV, and $41 \%$ from distributed solar. For the residential solar market, even though California and all major Northeast markets experienced annual contractions, 25 of 44 states saw growth in annual residential PV installations [3].

The other major change happening in the distribution grid is the implementation of demand response, which allows loads to adjust their consumption according to either direct control signals from the utility [4] or price signals [5]. Further, with the rapid development of smart appliances and home automation, in-house home energy management systems are developed that operate appliances based on customers own control objective [6] [7].

To better manage distribution-level resources while maintaining system reliability and stability, most advanced distribution energy management systems propose to implement distribution system state estimation (DSSE) for real-time applications. Weighted least-square (WLS) estimators are the most popular for DSSE [8]. There are voltage-based WLS and branch current-based WLS according to the selection of state variables. The algorithm in [9] has a better computation efficiency because it has a constant measurement Jacobian matrix at each iteration. Dynamic DSSE methods [10] [11] generally apply a Kalman filter to obtain a priori estimate, which is combined with latest measurements to forecast the system state. This method is based on several measurement snapshots in a time sequence.

The biggest challenge for DSSE comes from the lack of sensors [12]. Unlike the supervisory control and data acquisition (SCADA) systems in transmission grids, distribution systems normally do not have full observability from the SCADA systems, which roughly speaking means that the number of measurements is strictly less than that of the quantities being estimated. Also, measurements in distribution systems are truly heterogeneous. Bus voltage, branch current, power flows and switch status are measured from SCADAs, smart meters, autonomous switches, distribution synchrophasor units and intelligent electronics devices (e.g., inverters) in different time resolutions.

Several approaches in the literature tackle the issue of 
low observability. The works [13-16] proposed to improve observability by optimizing the minimal number of locations needed to perform the state estimation task. In [17], smart meter data was used to enhance observability of the system by solving the power flow over several consecutive time instances. Pseudo measurements are normally used to solve the mathematically underdetermined problem in DSSE because of the limited number of measurements [18-20].

The advances in machine learning have allowed for techniques such as neural networks to be used for state estimation [19, 21, 22]. Although machine learning techniques obtain accurate estimation results, they require a significant amount of historical data to train the algorithm, and this data might not be available.

Matrix completion is a well-known method in signal processing for the estimation of missing values within matrices [23]. Prior attempts to apply the algorithm to power systems include $[24,25]$, which perform load forecasting while accounting for missing data. Both of these approaches transform the data in a way that it is suitable for use with the matrix completion algorithm.

In this paper, we propose applying the matrix completion algorithm to estimate voltage phasors in low-observability distribution networks. To improve upon the algorithm's application to power systems, power flow equations are added as constraints to the problem formulation.

This approach is advantageous compared to previous works due to the large variety of situations in which it can be applied. While many works, such as [17, 20, 26], require voltage and power measurements to perform their estimations, this approach can make use of any quantity measured in the field. In this way, the algorithm can be used for a larger number of systems. Additionally, while methods such as [17] require collecting data over large time windows, this matrix completion approach is capable of estimating the state with a single time instance.

The paper is structured as follows. Section 2 overviews matrix completion and its implementation. Section 3 discusses how to apply this algorithm to solve DSSE and the modifications that were made. Section 4 demonstrates illustrative numerical results of the algorithm and Section 5 provides conclusion of the work.

\section{Matrix Completion}

Matrix completion method has been developed to estimate missing values of partially observed matrix [23]. It has been broadly used in signal processing community, e.g., to complete missing pixels in images [27] and to detect sensor location with partial distance information [28]. The concept of matrix completion is, for a given low rank matrix with a set of known elements $\Omega$, to make use of its low rank nature to estimate the missing values. When a matrix is low rank, there is a direct connection between the row and column elements within the matrix. This connection can then be used to determine elements that are unknown. This does, however, rely on the assumption that the matrix actually is in fact low rank, and that there are enough known elements to find the connection.

The matrix completion algorithm is first defined formally. Consider the space of real-valued $n_{1} \times n_{2}$ matrices $\mathbb{R}^{n_{1} \times n_{2}}$. Let $\mathcal{I}:=\left\{1, \ldots, n_{1}\right\} \times\left\{1, \ldots, n_{2}\right\}$ denote the index set, so that $(i, j) \in \mathcal{I}$ represents an element's index. For any matrix $W \in \mathbb{R}^{n_{1} \times n_{2}}$ and a subset $\Omega \subseteq \mathcal{I}$, let $W_{\Omega}$ denote

$$
W_{\Omega}=\left\{\begin{array}{r}
W_{i, j},(i, j) \in \Omega \\
0, \text { otherwise. }
\end{array}\right.
$$

Define $M$ as the matrix containing all measurements and $M_{\Omega}$ as the observation matrix, where $\Omega$ is the set of known elements within the matrix. From [23], the matrix completion problem can then be formulated as a rank minimization problem:

$$
\begin{array}{ll}
\min _{X \in \mathbb{R}^{n_{1} \times n_{2}}} & \operatorname{rank}(\mathrm{X}) \\
\text { s.t. } & X_{\Omega}=M_{\Omega},
\end{array}
$$

However, it can be seen that the formulation of (2) is a non-convex, NP-Hard optimization problem to solve, therefore making it unsuitable for practical use. Thus, the original problem is relaxed to that of minimizing the nuclear norm:

where

$$
\begin{array}{ll}
\min _{X \in \mathbb{R}^{n_{1} \times n_{2}}} & \|X\|_{*} \\
\text { s.t. } & X_{\Omega}=M_{\Omega},
\end{array}
$$

$$
\|X\|_{*}=\sum_{i=1}^{n} \sigma_{i}(X),
$$

$\sigma_{i}(X)$ is the $i^{t h}$ singular value, and $n:=\min \left\{n_{1}, n_{2}\right\}$.

Due to the nature of the equality constraint, formulation (3) is highly susceptible to noise. To alleviate this, [29] proposed a robust algorithm which allows for the matrix completion algorithm to account for noise in the observations. The algorithm modifies the equality constraint in (3) to

$$
\left\|X_{\Omega}-M_{\Omega}\right\|_{F} \leq \delta
$$

where $\|X\|_{F}$ is the Frobenius norm of $X$ which is defined as

$$
\|X\|_{F}=\sqrt{\sum_{i=1}^{m} \sum_{j=1}^{n}\left|X_{i, j}\right|^{2}} .
$$

The value of $\delta$ has a significant impact on the accuracy of the estimations. Since the parameter $\delta$ corresponds to the amount of noise on the data, the estimation accuracy will improve as the value decreases as this is equivalent to the amount of noise in the signal decreasing. 


\section{Low-Observability DSSE}

In this section, we first define the notion of low observability. Then, the matrix completion algorithm is proposed and applied to DSSE.

\subsection{Power System Model}

For brevity, we consider a balanced distribution network with a single slack bus and $N P Q$ buses. Let $\mathcal{N}=$ $\{1, \ldots, N\}$ denote the set of the $P Q$ buses, and $\mathcal{L} \subseteq \mathcal{N} \times$ $\mathcal{N}$ denote the set of distribution lines. We note that the formulation proposed in this paper can be easily extended to the general multiphase setting with both wye and delta connections, as proposed, e.g., in [30].

\subsection{Low observability}

Consider a linear (or linearized) measurement model of the form

$$
y=H x+w
$$

where $y$ denotes the measurement vector, $x$ denotes the state vector and $w$ denotes the measurements error. Then observability can be defined as invertibility of $H^{T} H$, namely as the existence of the explicit least-square solution

$$
x=\left(H^{T} H\right)^{-1} H^{T} y .
$$

Therefore, we say that the system is under low-observability conditions whenever $H^{T} H$ is not full rank, at which point that conventional methods such as weighted least square are not directly applicable.

\subsection{Matrix Set-Up}

Since matrix completion relies on the assumption of low rank matrices, the choice of quantities with which the matrix is created has a significant impact on the accuracy of the results. The higher the link between the quantities and voltage, the more accurate the resulting estimation will be. However, as long as there is even a slight correlation between the quantity and voltage, the quantity is able to be used in the estimation algorithm.

In order to allow for the use of the largest number and variety of variables, we set up the matrix in terms of system connection lines rather than buses. In this way, each row of the matrix represents one line in the system, and each column represents one variable. The resultant matrix columns then are the real and reactive voltage from the source bus of the line; the total real and reactive power entering the line source bus from all sources (generators and lines); the real and reactive current flowing through the line; the load at the source bus; and the voltage magnitude at the source bus. Formally, for every line $(f, t) \in \mathcal{L}$, the corresponding row in the matrix $M$ is given by

$$
\begin{array}{r}
{\left[\operatorname{Re}\left(V_{f}\right), \operatorname{Im}\left(V_{f}\right), P_{\text {inf }_{f}}, Q_{\text {inf }_{f}}, \operatorname{Re}\left(I_{f, t}\right), \operatorname{Im}\left(I_{f, t}\right), P_{\text {flow }_{f, t}},\right.} \\
\left.Q_{\text {flow }_{f, t}}, P_{\text {load }_{f}}, Q_{\text {load }_{f}},\left|V_{f}\right|\right] .
\end{array}
$$

We note that the proposed algorithm is not limited to the variables chosen. In fact, any quantity which has a correlation to voltage can be used to supplement and improve the results. In this paper, the elements that will be included in the matrix and their correlation with state variables will be described in the follow section.

\subsection{Power Flow Constraints}

The standard matrix completion algorithm may be able to return estimates of the voltage under specific conditions, but the formulation can be significantly improved upon through the inclusion of power flow constraints. We next formulate several corresponding constraints to be included to the original formulation detailed in Section 2.

First, since the objective matrix contains both the voltages at each bus and the current flowing between buses, power flow constraints can be introduced to the optimization in a form of the following linear equality constraint:

$$
\left(V_{f}-V_{t}\right) Y_{f t}=I_{f t}, \forall(f, t) \in \mathcal{L} .
$$

One potential issue when including new constraints is ensuring the problem remains feasible. To avoid these potential issues and increase the robustness of the algorithm, the new constraints are relaxed and bounded by a tolerance thus becoming

$$
-\epsilon_{f, t} \leq\left(V_{f}-V_{t}\right) Y_{f t}-I_{f t} \leq \epsilon_{f, t}, \forall(f, t) \in \mathcal{L},
$$

where $\epsilon_{f, t}$ is the error tolerance for line $(f, t) \in \mathcal{L}$.

Next, it is a natural requirement for there to be net zero power at each bus. Thus, the power flowing into the bus must be equal to the power consumed at the bus in addition to the power leaving the bus. This can be formulated as

$$
\begin{gathered}
P_{i n_{f}}-\sum_{t \in \mathcal{N}} P_{\text {flow }_{f, t}}-P_{\text {load }_{f}}=0, \forall f \in \mathcal{N}, \\
Q_{\text {in }_{f}}-\sum_{t \in \mathcal{N}} Q_{\text {flow }_{f, t}}-Q_{\text {load }_{f}}=0, \forall f \in \mathcal{N} .
\end{gathered}
$$

Similarly to constraint (10), we relax these equality constraints as follows:

$$
\begin{gathered}
-\tau_{f} \leq P_{\text {in }_{f}}-\sum_{t \in \mathcal{N}} P_{\text {flow }_{f, t}}-P_{\text {load }_{f}} \leq \tau_{f}, \forall f \in \mathcal{N} \\
-\tau_{f} \leq Q_{\text {in }_{f}}-\sum_{t \in \mathcal{N}} Q_{\text {flow }_{f, t}}-Q_{\text {load }_{f}} \leq \tau_{f}, \forall f \in \mathcal{N}
\end{gathered}
$$


where $\tau_{f}$ is the error tolerance for bus $f \in \mathcal{N}$. Feasibility can always be ensured by selecting tolerance values which create a feasible problem. However, since the accuracy of the resultant estimation is dependent on the tolerance being minimal, the values used need to be included in the optimization objective. Therefore, augmenting the original (robust) matrix completion problem (3), (5) with the power flow constraints, results in the following optimization problem:

$$
\min _{X \in \mathbb{R}^{n_{1} \times n_{2}},\left\{\epsilon_{f, t}\right\},\left\{\tau_{f}\right\}}\|X\|_{*}+w_{1} \sum_{(f, t) \in \mathcal{L}} \epsilon_{f, t}+w_{2} \sum_{f \in \mathcal{N}} \tau_{f}
$$

$$
\begin{array}{ll}
\text { s.t. } & \left\|X_{\Omega}-M_{\Omega}\right\|_{F} \leq \delta \\
& (10),(12) \\
& \epsilon_{f, t} \geq 0, \forall(f, t) \in \mathcal{L} \\
& \tau_{f} \geq 0, \forall f \in \mathcal{N},
\end{array}
$$

where $w_{1}, w_{2}>0$ are weighting parameters; $n_{1}:=|\mathcal{L}|$ is the number of lines in the network; and $n_{2}$ is the number of variables used for estimation. Observe that, since the measurements are in rectangular coordinates, (13) is a convex optimization problem and hence can be solved efficiently.

We note that, when the measurements of voltage magnitudes and/or power injections are available, formulation (13) can be augmented with additional constraints to capture the dependence between these variables.

The LinDistFlow approximation for power-flow equations [31] can be used under the assumption that the network is radial and of low power, as found in distribution networks. The LinDistFlow voltage approximation is given by

$$
\left|V_{t}\right|=\left|V_{f}\right|-\left(r_{f, t} P_{\text {flow }_{f, t}}+x_{f, t} Q_{\text {flow }_{f, t}}\right) /\left|V_{0}\right|
$$

where $V_{0}$ is the voltage magnitude at the feeder head, and $r_{f, t}$ and $x_{f, t}$ are the resistance and reactance of line $(f, t)$, respectively. This constraint is once again relaxed and added to the optimization problem, resulting in:

$$
\begin{aligned}
& \min _{X \in \mathbb{R}^{n_{1} \times n_{2}},\left\{\epsilon_{f, t}\right\},\left\{\tau_{f}\right\},\left\{\gamma_{f, t}\right\}}\|X\|_{*} \\
& \quad+w_{1} \sum_{(f, t) \in \mathcal{L}} \epsilon_{f, t}+w_{2} \sum_{f \in \mathcal{N}} \tau_{f}+w_{3} \sum_{(f, t) \in \mathcal{L}} \gamma_{f, t} \\
& \text { s.t. }(13 \mathrm{~b})-(13 \mathrm{e}) \\
& \quad|| V_{t}|-| V_{f} \mid+\left(r_{f, t} P_{f l o w_{f, t}}\right. \\
& \left.\quad+x_{f, t} Q_{f l o w_{f, t}}\right) /\left|V_{0}\right| \mid \leq \gamma_{f, t}, \forall(f, t) \in \mathcal{L} \\
& \gamma_{f, t} \geq 0, \forall(f, t) \in \mathcal{L}
\end{aligned}
$$

All the tunning parameters are decided through trial and error.

\section{Simulation and Results}

The test case for this work is the IEEE standard 33 bus system as shown in Figure 1. The system parameters are chosen specifically to demonstrate the accuracy of the proposed DSSE method over a large range of voltage values, therefore the voltage profile range $(1$ p.u. $\quad-0.82$ p.u.) in the system does not reflect a normal distribution voltage profile (see Figure 2). Unless otherwise stated, all simulations are run under the assumption of a single voltage phasor measurement and $50 \%$ of the remaining quantities. We acknowledge that the test system is a three-phase balanced distribution system. However the developed method can be easily scaled to solve unbalanced system by utilizing the linear power multiphase model in [30]

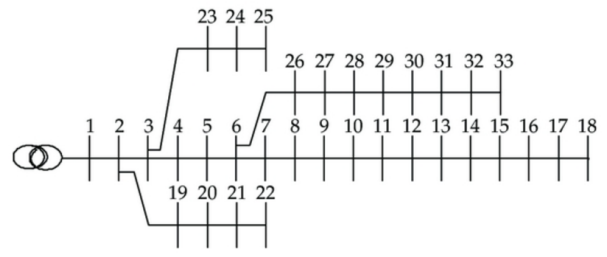

Figure 1. IEEE Standard 33 Bus System

Figure 2 shows the results of applying the matrix completion formulation (15) to the entire IEEE 33 bus radial system.

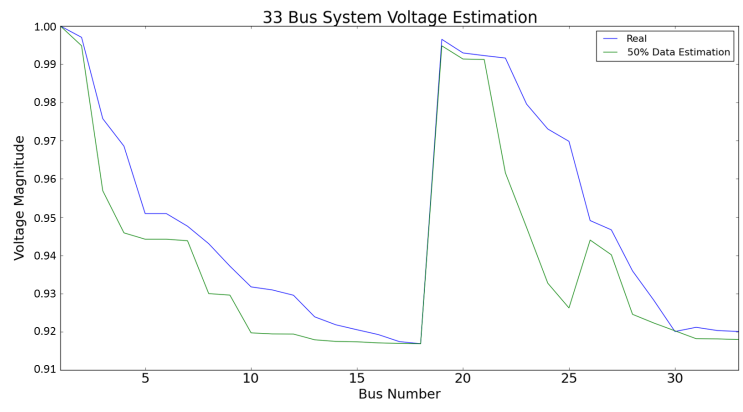

Figure 2. Voltage Estimation of Radial Network

It can be seen that the results of the estimation are accurate, following the trend of the system properly. There are occasional large deviations from the true value due to the high dependence on which data was available to make the estimation. These results were produced under the assumption that the data available was uniformly randomly sampled from the complete data set, which is not necessarily the case for real systems.

Figure 3 shows the impact of observability on the mean absolute percentage error(MAPE) of the estimations. 


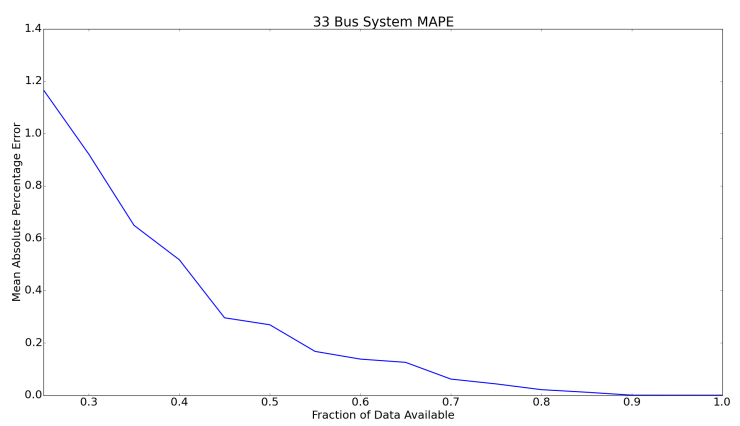

Figure 3. MAPE as Observability Increases

The results in Figure 4 show that the approach is possible for angle estimations as well, with a slight increase on the noise of the estimations. Additionally, the amount of voltage measurements required to obtain accurate results is increased when estimating angle values. The plot in Figure 4 was obtained with a voltage phasor measurement at Bus 1 and the ending Bus of all branches $(18,22,25,33)$ in the IEEE 33 Bus system.

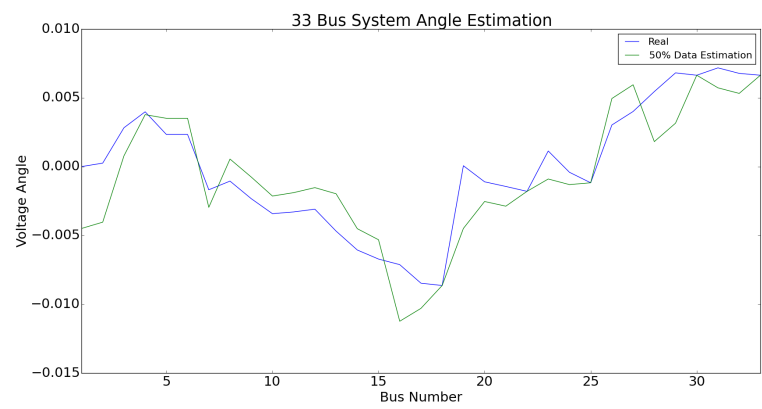

Figure 4. Voltage Angle Estimation of Radial Network

While this approach results in accurate estimations, no algorithm would be acceptable without being able to handle error on the measurements. To show that this procedure is robust against system errors, varying amounts of noise were added to the data. All plots were run using 50 percent data availability, while only a single voltage measurement was used at bus 1 . Additionally, the missing elements were the same for each case such that the only difference in estimations was the error.

From Figure 5 it can be seen that while measurement errors do have some impact on the resultant estimation, the availability of data has a much more significant impact. The presence of data or lack thereof will determine the trend of the estimations, while the differences in error only cause an offset in the final estimations. Since the estimations are done based upon the value of the measurement, rather than the type of measurement, errors from one parameter, such as voltage angle, will effect all other parameters as well.

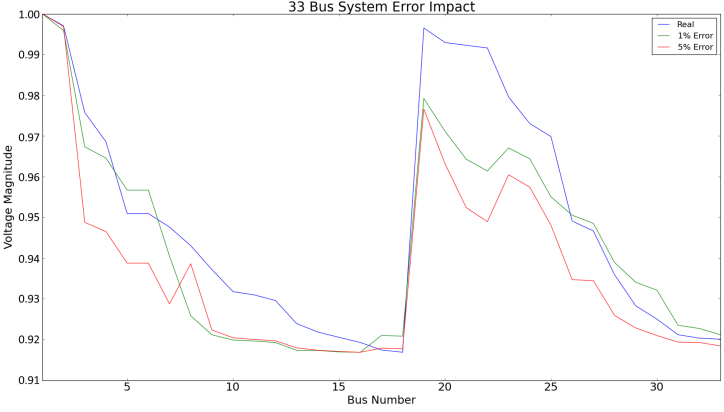

Figure 5. Impact of Measurement Errors

\section{Conclusion}

Due to the rapid growth in distributed generation and active load participation in system operation, the need for state estimation in distribution networks can no longer be ignored. However, the issue of scale and observability has not gone away; thus it has become necessary to be able to perform state estimation in systems that have low observability using heterogeneous measurements input.

In this paper, a matrix completion approach is detailed which makes use of supplemental measurements from smart meters, distributed resources, and any data which are available to perform state estimation. The traditional matrix completion algorithm is improved through the inclusion of power flow constraints which allows for accurate results in the estimation while required less data than previously. Through the estimation of voltage phasors, it is shown that this approach obtains relatively accurate results under various scenarios of low observability. The method theoretically should work for a low rank matrix. From the numerical tests, the data matrix is verified to be approximately low-rank (there are few singular values that are close to zero). This depends on the accuracy provided by the linear power flow model that presented in [31].

As described at the beginning of the paper, the presented method will work with any type of measurements. Currently, the AMI data are assumed to be aggregated at the primary side of the distribution transformer because the secondary system topology and parameters are not always available. However, some proposed work [32] uses AMI data to estimate secondary systems should work nicely with the matrix completion method for state estimation. The authors will pursue such knowledge in the following works.

\section{Acknowledgement}

This work was authored in part by Alliance for Sustainable Energy, LLC, the Manager and Operator of the National Renewable Energy Laboratory for the U.S. Department of Energy (DOE) under Contract No. 
DE-AC36-08GO28308. Funding provided by U.S. Department of Energy Office of Energy Efficiency and Renewable Energy Solar Energy Technologies Office. The views expressed in the article do not necessarily represent the views of the DOE or the U.S. Government. The U.S. Government retains and the publisher, by accepting the article for publication, acknowledges that the U.S. Government retains a nonexclusive, paid-up, irrevocable, worldwide license to publish or reproduce the published form of this work, or allow others to do so, for U.S. Government purposes.

\section{References}

[1] D. Salem-Natarajan, L. Zhao, W. Shao, M. Varghese, S. Ghosh, M. Subramanian, G. Lin, H.-D. Chiang, and H. Li, "State estimator for CA ISO market and security applications-relevance and readiness," in IEEE Power and Energy Society General Meeting-Conversion and Delivery of Electrical Energy in the 21st Century, 2008.

[2] Solar Energy Industries Association and others, "US solar market insight," 2016.

[3] Solar Energy Industries Association and others, "Solar market insight report 2017," SEIA. org, 2018.

[4] H. Jorge, C. H. Antunes, and A. G. Martins, "A multiple objective decision support model for the selection of remote load control strategies," IEEE Transactions on Power Systems, vol. 15, no. 2, pp. 865-872, 2000.

[5] L. Xie, J.-Y. Joo, and M. D. Ilić, "Integration of intermittent resources with price-responsive loads," in North American Power Symposium (NAPS), 2009.

[6] Z. Yu, L. Jia, M. C. Murphy-Hoye, A. Pratt, and L. Tong, "Modeling and stochastic control for home energy management," IEEE Transactions on Smart Grid, vol. 4, no. 4 , pp. 2244-2255, 2013.

[7] R. Yang and Y. Zhang, "Coordinated optimization of distributed energy resources and smart loads in distribution systems," in Power and Energy Society General Meeting (PESGM), July 2016.

[8] A. Primadianto and C.-N. Lu, "A review on distribution system state estimation," IEEE Transactions on Power Systems, vol. 32, no. 5, pp. 3875-3883, 2017.

[9] M. E. Baran and A. W. Kelley, "State estimation for real-time monitoring of distribution systems," IEEE Transactions on Power Systems, vol. 9, no. 3, pp. 1601-1609, 1994.

[10] C. Carquex, "State estimation in power distribution systems," Master's thesis, University of Waterloo, 2017.

[11] S. Sarri, M. Paolone, R. Cherkaoui, A. Borghetti, F. Napolitano, and C. A. Nucci, "State estimation of active distribution networks: comparison between wls and iterated kalman-filter algorithm integrating pmus," in 3rd IEEE PES International Conference and Exhibition on Innovative Smart Grid Technologies (ISGT Europe), 2012.

[12] M. E. Baran, "Challenges in state estimation on distribution systems," in 2001 Power Engineering Society Summer Meeting. Conference Proceedings (Cat. No.01CH37262), vol. 1, pp. 429-433 vol.1, July 2001.

[13] M. E. Baran, J. Zhu, and A. W. Kelley, "Meter placement for real-time monitoring of distribution feeders," in Proceedings of Power Industry Computer Applications Conference, pp. 228-233, May 1995.
[14] A. Shafiu, N. Jenkins, and G. Strbac, "Measurement location for state estimation of distribution networks with generation," IEE Proceedings - Generation, Transmission and Distribution, vol. 152, pp. 240-246, March 2005.

[15] R. Singh, B. C. Pal, and R. B. Vinter, "Measurement placement in distribution system state estimation," IEEE Transactions on Power Systems, vol. 24, pp. 668-675, May 2009.

[16] M. Barukčić, M. Vukobratović, D. Masle, D. Buljić, and $\breve{Z}$. Herderić, "The evolutionary optimization approach for voltage profile estimation in a radial distribution network with a decreased number of measurements," in 15th International Conference on Electrical Machines, Drives and Power Systems (ELMA), pp. 26-31, June 2017.

[17] S. Bhela, V. Kekatos, and S. Veeramachaneni, "Enhancing observability in distribution grids using smart meter data," IEEE Transactions on Smart Grid, vol. PP, no. 99, 2017.

[18] K. A. Clements, "The impact of pseudo-measurements on state estimator accuracy," in IEEE Power and Energy Society General Meeting, July 2011.

[19] E. Manitsas, R. Singh, B. C. Pal, and G. Strbac, "Distribution system state estimation using an artificial neural network approach for pseudo measurement modeling," IEEE Transactions on Power Systems, vol. 27, pp. 1888-1896, Nov. 2012.

[20] J. Wu, Y. He, and N. Jenkins, "A robust state estimator for medium voltage distribution networks," IEEE Transactions on Power Systems, vol. 28, pp. 1008-1016, May 2013.

[21] M. Pertl, K. Heussen, O. Gehrke, and M. Rezkalla, "Voltage estimation in active distribution grids using neural networks," in IEEE Power and Energy Society General Meeting (PESGM), July 2016.

[22] H. Jiang and Y. Zhang, "Short-term distribution system state forecast based on optimal synchrophasor sensor placement and extreme learning machine," in IEEE Power and Energy Society General Meeting (PESGM), July 2016.

[23] E. J. Candès and B. Recht, "Exact matrix completion via convex optimization," Foundations of Computational Mathematics, vol. 9, pp. 717-772, Apr. 2009.

[24] S. J. Kim and G. B. Giannakis, "Load forecasting via low rank plus sparse matrix factorization," in Asilomar Conference on Signals, Systems and Computers, pp. 1682-1686, Nov. 2013.

[25] J. A. Bazerque and G. B. Giannakis, "Nonparametric basis pursuit via sparse kernel-based learning: A unifying view with advances in blind methods," IEEE Signal Processing Magazine, vol. 30, pp. 112-125, July 2013.

[26] C. Klauber and H. Zhu, "Distribution system state estimation using semidefinite programming," in North American Power Symposium (NAPS), Oct. 2015.

[27] H. Ji, C. Liu, Z. Shen, and Y. Xu, "Robust video denoising using low rank matrix completion," in IEEE Conference on Computer Vision and Pattern Recognition (CVPR), pp. 1791-1798, 2010.

[28] Y. Ding, N. Krislock, J. Qian, and H. Wolkowicz, "Sensor network localization, euclidean distance matrix completions, and graph realization," Optimization and Engineering, vol. 11, no. 1, pp. 45-66, 2010.

[29] E. J. Candes and Y. Plan, "Matrix completion with noise," Proceedings of the IEEE, vol. 98, pp. 925-936, June 2010.

[30] A. Bernstein, C. Wang, E. Dall'Anese, J.-Y. Le Boudec, and C. Zhao, "Load-flow in multiphase distribution networks: Existence, uniqueness, and linear models," 2017. [Online] Available at: http://arxiv.org/abs/1702.03310. 
[31] K. Turitsyn, P. Sulc, S. Backhaus, and M. Chertkov, "Local control of reactive power by distributed photovoltaic generators," in First IEEE International Conference on Smart Grid Communications, pp. 79-84, Oct. 2010.

[32] J. Peppanen, C. Rocha, J. A. Taylor, and R. C. Dugan, "Secondary low-voltage circuit modelshow good is good enough?," IEEE Transactions on Industry Applications, vol. 54, no. 1, pp. 150-159, 2018. 